\title{
Optimum Coupling Coefficients in Second-Order Series-Coupled Ring Resonator for Nonblocking Wavelength Channel Switch
}

\author{
Tomoyuki Kato, Student Member, IEEE, and Yasuo Kokubun, Senior Member, IEEE
}

\begin{abstract}
This paper analytically derived the optimum condition of coupling efficiency for the lossy double ring resonator. Using this condition, a new concept of nonblocking wavelength switch using a series-coupled ring resonator is proposed. The crosstalk characteristics of the wavelength switch were investigated using the transfer matrix. Using this optimum condition, the crosstalk of the wavelength switch can be reduced.
\end{abstract}

Index Terms-Microresonators, microring resonators, optical waveguide filers, transfer function matrices, wavelength division multiplexing.

\section{INTRODUCTION}

$\mathbf{M}$ ICRORING resonators can create a box-like spectrum response shape by synthesizing them [1], [2]. The series-coupled microring resonator filter shown in Fig. 1 is advantageous to an add/drop multiplexer since it can achieve a box-like spectrum response by optimizing coupling efficiencies between the bus line and the resonator and between resonators. In addition, by controlling the resonant wavelengths of individual resonators independently, a nonblocking wavelengthchannel-selective switch can be realized [3].

The switching characteristics such as extinction ratio, crosstalk, and insertion loss, however, depend on coupling efficiencies between the bus line and the ring and between ring resonators and propagation loss in the resonator. The most useful property of a series-coupled microring resonator is that the lossless box-like filter response can be obtained in contradiction to flat-top arrayed waveguide grating (AWG) filters. Although this condition was obtained by Little et al. [4], it was obtained assuming that the propagation loss of the ring resonator is negligibly small.

However, the microring resonator suffers radiation, scattering, and absorption losses, and the optimum condition of a flat passband for the lossy ring resonator was not made clear. The loss in the resonator deforms the spectrum response shape, and the optimum condition of coupling efficiencies depends on the

Manuscript received May 24, 2005; revised September 17, 2005. This work was supported in part by a Grant-in-Aid for Scientific Research on Priority Areas No. 13026210 from the Ministry of Education, Culture, Sports, Science and Technology, the Futaba Electronics Memorial Foundation, and the 21st Century COE Program in Ministry of Education, Culture, Sports, Science and Technology.

The authors are with Department of Electrical and Computer Engineering, Graduate School of Engineering, Yokohama National University, Hodogaya-ku, Yokohama 240-8501, Japan(e-mail: kato@koklab.seg.ynu.ac.jp; kokubun@ynu.ac.jp).

Digital Object Identifier 10.1109/JLT.2005.862425

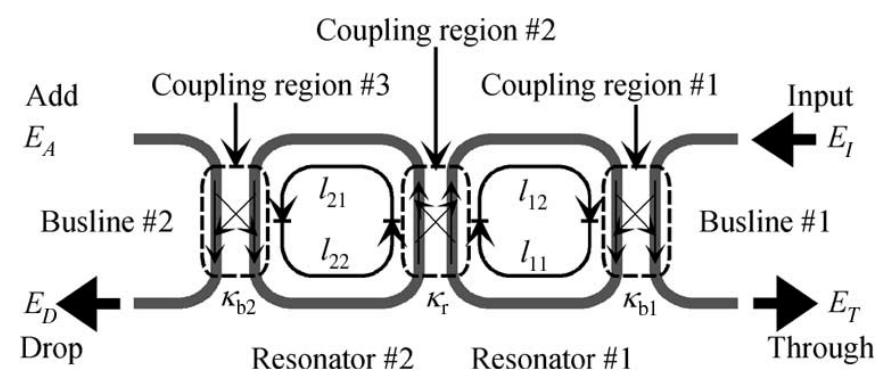

Fig. 1. Second-order series-coupled ring resonator.

loss in the resonator. Although the critical coupling condition was reported for an all-pass single-ring resonator with only input and output ports [5], [6], the wavelength switch proposed in this paper requires add and drop ports; thus, this condition is not applicable.

In this study, we investigated the optimum condition of coupling efficiencies (so-called Butterworth condition) and made clear that the optimum condition for the lossy case is quite different from that for the lossless case. In this analysis, the coupling efficiency between the input bus line and the ring resonator is identical to that between the output bus line and the ring resonator. Therefore, only two coupling efficiencies are free to be chosen.

If the resonator suffers a loss, a small amount of light is transmitted to the through port at the resonant frequency under the conventional maximally flat condition, and the crosstalk is deteriorated. However, our optimum condition can reduce transmittance to the through port to zero at the resonant frequency and the crosstalk of the wavelength switch can be improved.

\section{Transfer Function of Double Ring Resonator}

The transfer function of a series-coupled ring resonator can be derived using transfer matrix [6]-[9] as follows. The seriescoupled ring resonator consists of coupling regions between waveguides and recursive delay lines as shown in Fig. 1. If no loss occurs at the coupling region and the waveguides are matched, i.e., the propagation constants of coupled waveguides are equal, the field amplitudes at the output ports of the coupling region as shown in Fig. 2(a) are expressed by

$$
\left[\begin{array}{l}
E_{c 1}^{\prime} \\
E_{c 2}^{\prime}
\end{array}\right]=\left[\begin{array}{cc}
\sqrt{t} & -j \sqrt{\kappa} \\
-j \sqrt{\kappa} & \sqrt{t}
\end{array}\right]\left[\begin{array}{l}
E_{c 1} \\
E_{c 2}
\end{array}\right]
$$




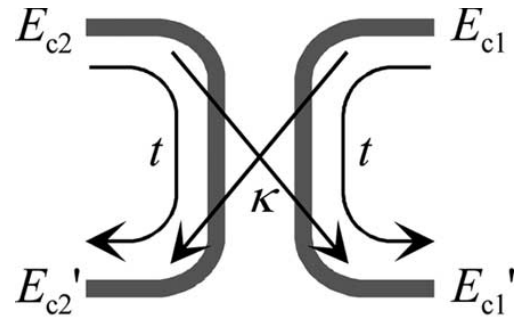

(a)

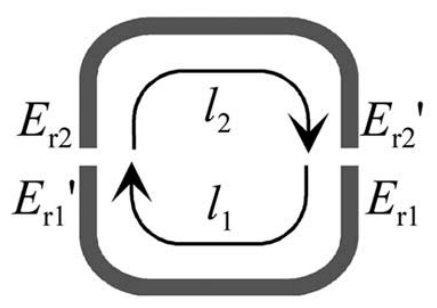

(b)

Fig. 2. Components of ring resonator. (a) Coupling region. (b) Recursive delay line.

where $t$ is the power transmittance and $\kappa$ is the power coupling efficiency of the coupler. If the coupler is lossless, $\kappa=1-t$ and the matrix is unitary. Equation (1) can be rewritten as the relation between $\left(E_{c 2}, E_{c 2}^{\prime}\right)$ and $\left(E_{c 1}, E_{c 1}^{\prime}\right)$ by

$$
\begin{aligned}
{\left[\begin{array}{l}
E_{c 2} \\
E_{c 2}^{\prime}
\end{array}\right] } & =\mathbf{C}\left[\begin{array}{l}
E_{c 1} \\
E_{c 1}^{\prime}
\end{array}\right] \\
\mathbf{C} & =\frac{j}{\sqrt{1-t}}\left[\begin{array}{cc}
-\sqrt{t} & 1 \\
-1 & \sqrt{t}
\end{array}\right] .
\end{aligned}
$$

The propagation in the ring resonator shown in Fig. 2(b) is expressed by

$$
\begin{aligned}
{\left[\begin{array}{c}
E_{r 2} \\
E_{r 1}^{\prime}
\end{array}\right] } & =\mathbf{R}\left[\begin{array}{c}
E_{r 2}^{\prime} \\
E_{r 1}
\end{array}\right] \\
\mathbf{R} & =\left[\begin{array}{cc}
{\sqrt{a_{2}}}^{-1} \exp \left(j \beta l_{2}\right) & 0 \\
0 & \sqrt{a_{1}} \exp \left(-j \beta l_{1}\right)
\end{array}\right]
\end{aligned}
$$

where $\beta$ is the propagation constant and $a_{i}$ represents the transmittance after transmission of length $l_{i}(i=1$ or 2$)$ in the resonator. $\mathbf{R}$ represents the clockwise propagation and $\mathbf{R}^{-1}$ represents the counter-clockwise propagation.

Cascading (2)-(5) at each stage, the transfer matrix of a second-order series-coupled ring resonator is given by

$$
\left[\begin{array}{c}
E_{D} \\
E_{A}
\end{array}\right]=\mathbf{C}_{b 2} \mathbf{R}_{r 2}^{-1} \mathbf{C}_{r 1} \mathbf{R}_{r 1} \mathbf{C}_{b 1}\left[\begin{array}{c}
E_{T} \\
E_{I}
\end{array}\right] .
$$

From (6), the transfer function from input port to through port $E_{T} / E_{I}$ and the transfer function from input port to drop port $E_{D} / E_{I}$ are obtained as

$$
\begin{aligned}
\frac{E_{T}}{E_{I}} & =\frac{a \sqrt{t_{b}} e^{-2 j \beta L}-\sqrt{a}\left(1+t_{b}\right) \sqrt{t_{r}} e^{-j \beta L}+\sqrt{t_{b}}}{a t_{b} e^{-2 j \beta L}-2 \sqrt{a t_{b} t_{r}} e^{-j \beta L}+1} \\
\frac{E_{D}}{E_{I}} & =\frac{\sqrt{a}\left(1-t_{b}\right) \sqrt{1-t_{r}} e^{-j \beta L}}{a t_{b} e^{-2 j \beta L}-2 \sqrt{a t_{b} t_{r}} e^{-j \beta L}+1}
\end{aligned}
$$

where $t_{r}$ is the power transmittance of the coupling region between ring resonators, $t_{b}$ is that between bus line and ring waveguides, and $a$ is the transmittance per round. We assumed that $\mathbf{C}_{b 1}=\mathbf{C}_{b 2}$, i.e., $t_{b}=t_{b 1}=t_{b 2}, l_{11}=l_{12}=l_{21}=l_{22}=$ $L / 2$, and $a_{11}=a_{12}=a_{21}=a_{22}=a^{1 / 2}$.

The power transmittance to the through port $P_{T}=\left|E_{T} / E_{I}\right|^{2}$ is given by

$$
P_{T}=\frac{A\left[\left(d_{+} \sqrt{k_{n}}-\cos (\beta L)\right)^{2}+d_{-}\left(1-d_{+} k_{n}\right)\right]}{1+A\left(\sqrt{k_{d}}-\cos (\beta L)\right)^{2}}
$$

where

$$
\begin{aligned}
A & =\frac{4 a t_{b}}{\left(1-a t_{b}\right)^{2}\left(1-t_{r}\right)} \\
k_{n} & =\frac{a\left(1+t_{b}\right)^{2} t_{r}}{(1+a)^{2} t_{b}} \\
d_{ \pm} & =\frac{(1 \pm a)^{2}}{4 a} \\
k_{d} & =\frac{\left(1+a t_{b}\right)^{2} t_{r}}{4 a t_{b}} .
\end{aligned}
$$

On the other hand, the power transmittance to the drop port $P_{D}=\left|E_{D} / E_{I}\right|^{2}$ is given by

$$
P_{D}=\frac{p}{1+A\left(\sqrt{k_{d}}-\cos (\beta L)\right)^{2}}
$$

where

$$
p=\frac{a\left(1-t_{b}\right)^{2}}{\left(1-a t_{b}\right)^{2}} .
$$

\section{Optimum Condition}

The peak in the drop port response and the notch in the through port response may split into the number of ring resonators in the series-coupled ring resonator even if the ring resonators are identical. When the ratio of coupling efficiencies $\kappa_{r} / \kappa_{b}$ is larger than a certain condition discussed later, the passband has two peaks, i.e., the passband involves a ripple. When the ratio of coupling efficiencies $\kappa_{r} / \kappa_{b}$ is small, the passband has a single peak, although the peak transmittance decreases. Thus, the optimization of coupling efficiencies is required for a box-like spectrum response.

\section{A. Through Port Response}

From (9), the shape of the through port response at the resonant wavelength varies depending on coupling efficiencies. Fig. 3 shows the transmittance around resonant wavelength in the case of $a=1.0(0 \mathrm{~dB}), \kappa_{b}=0.2$, and the parameter is $\kappa_{r}$. At a certain coupling efficiency, the transmittance at resonant wavelength is reduced to almost zero $(-\infty \mathrm{dB})$, which corresponds to the critical coupling of an all-pass filter [6]. Thus, we define this condition as the optimum condition for 


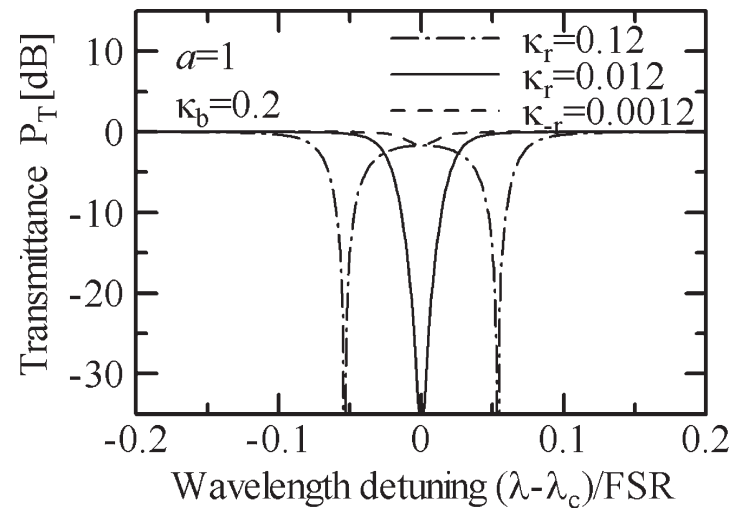

Fig. 3. Through port spectrum response.

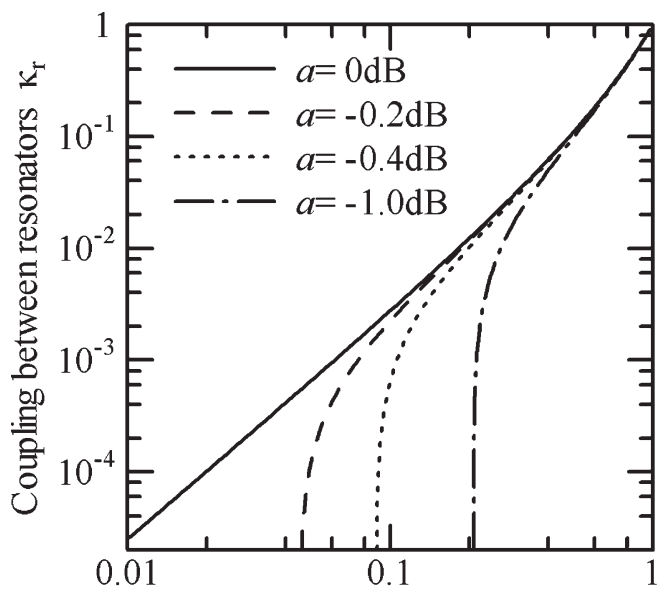

Coupling between busline and resonator $\kappa_{\mathrm{b}}$

Fig. 4. Optimum coupling efficiencies for through port response.

through port response. Then, the through port response has a sharp notch.

This condition is equivalent to the case that the numerator of (9) is equal to zero at resonant wavelength. Let us define $t_{r t}$ as the optimum $t_{r}$ for through port under this condition. Then the relational expression between $t_{b}$ and $t_{r}$ is expressed by

$$
t_{r t}=\frac{(1+a)^{2} t_{b}}{a\left(1+t_{b}\right)^{2}}
$$

From this condition, the optimum condition of coupling efficiency is expressed as

$$
\kappa_{r t}=\frac{\left[a-\left(1-\kappa_{b}\right)\right]\left[1-a\left(1-\kappa_{b}\right)\right]}{a\left(2-\kappa_{b}\right)^{2}}
$$

where $\kappa_{r t}$ is the optimum $\kappa_{r}$, and $\kappa=1-t$. The relation in (17) is illustrated in Fig. 4. In the case of lossless and weak $\kappa_{b}, \kappa_{r t} \sim\left(\kappa_{b} / 2\right)^{2}$. When the resonator involves loss, $\kappa_{b}$ has a threshold value for optimum condition.

Since $0<\kappa_{r t}<1, \kappa_{b}$ has to be in the range

$$
1-a<\kappa_{b}<1
$$

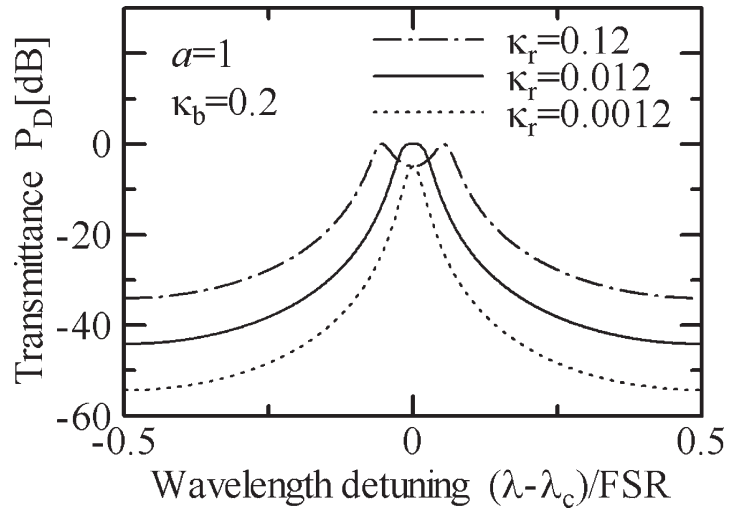

Fig. 5. Drop port response.

under this optimum condition, and the transfer function is rewritten as

$$
P_{T o}=\frac{A_{T}(1-\cos (\beta L))^{2}}{1+A_{T}\left(k_{d t}-\cos (\beta L)\right)^{2}}
$$

where

$$
\begin{aligned}
A_{T} & =\frac{4 a^{2} t_{b}\left(1+t_{b}\right)^{2}}{\left(1-a t_{b}\right)^{3}\left(a-t_{b}\right)} \\
k_{d t} & =\frac{(1+a)\left(1+a t_{b}\right)}{2 a\left(1+t_{b}\right)} .
\end{aligned}
$$

\section{B. Drop Port Response}

From (14), the shape of the peak at resonant wavelength varies according to coupling efficiencies. Fig. 5 shows the transmittance of drop port around resonant wavelength in the case of $a=1.0(0 \mathrm{~dB}), \kappa_{b}=0.2$, and the parameter is $\kappa_{r}$. In a certain coupling efficiency, the peak is maximally flat. Thus, we define this condition as the optimum condition for the Butterworth response of drop port.

This condition is that the second-order derivative of (14) with respect to $\phi=\beta L$ is equal to zero at resonant wavelength. Let us define the flatness of peak by

$$
\begin{aligned}
\text { Flatness } & =\left.\frac{d^{2} P_{D}}{d \phi^{2}}\right|_{\phi=0} \\
& =\frac{2 p A\left(1-k_{d}\right)}{\left[1+A\left(k_{d}-1\right)^{2}\right]^{2}} .
\end{aligned}
$$

When this value is positive, the spectrum response has two peaks. When this value is negative, the spectrum response has a single peak. When this value is zero, the peak is maximally flat.

The optimum condition is that the numerator of (22) is equal to zero. Let us define $t_{r d}$ as the optimum $t_{r}$ for the drop port under this condition. The relational expression between $t_{b}$ and $t_{r d}$ is expressed by

$$
t_{r d}=\frac{4 a t_{b}}{\left(1+a t_{b}\right)^{2}} .
$$




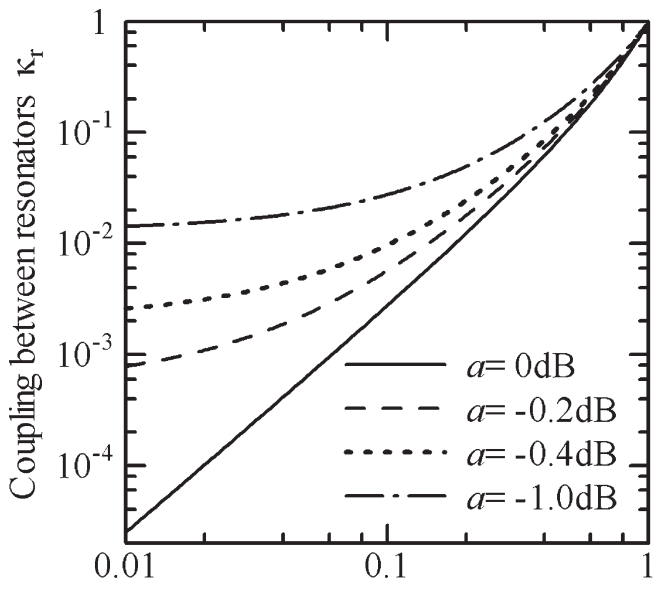

Coupling between busline and resonator $\kappa_{\mathrm{b}}$

Fig. 6. Optimum coupling efficiencies from drop port response.

From this condition, the optimum condition of coupling efficiency is expressed by

$$
\kappa_{r d}=\left[\frac{1-a\left(1-\kappa_{b}\right)}{1+a\left(1-\kappa_{b}\right)}\right]^{2}
$$

where $\kappa_{r d}$ is the optimum $\kappa_{r}$ for this condition. The relation of (24) is also shown in Fig. 6. Compared with the condition in [4], which is $\kappa_{r}=\kappa_{b}^{2} / 4$, these two conditions are different in the lossy or strong coupling case. In the lossless case, this condition is equal to the optimum condition of through port response. When the resonator involves loss, $\kappa_{r}$ has a threshold value for optimum condition.

Since $0<\kappa_{b}<1, \kappa_{r}$ has to be in the range

$$
\left[\frac{1-a}{1+a}\right]^{2}<\kappa_{r}<1
$$

under this optimum condition, and the transfer function is rewritten as

$$
P_{D o}=\frac{p}{1+A_{d}(1-\cos (\beta L))^{2}}
$$

where

$$
A_{d}=\frac{4 a t_{b}}{\left(1-a t_{b}\right)^{4}} .
$$

\section{Spectrum Shape of Double Ring Resonator}

\section{A. Through Port Response}

The spectrum response shape of a second-order seriescoupled ring resonator is determined by the ratio of coupling efficiencies. So the transmittance of the coupling region between resonators is expressed by the transmittance of the coupling region between bus line and ring waveguides under the optimum condition for through port response as

$$
t_{r}=k_{n} \frac{(1+a)^{2} t_{b}}{a\left(1+t_{b}\right)^{2}}
$$

Wavelength $\lambda$

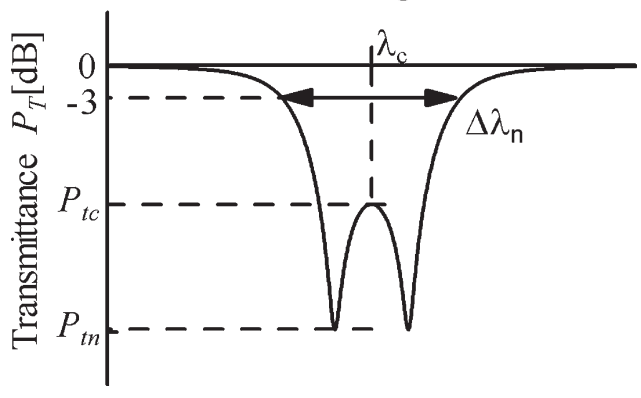

Fig. 7. Spectrum shape of through port response.

where $k_{n}$ is the ratio of $t_{r}$ to $t_{r t}$. Since $0<t_{r}<1$, the range of $k_{n}$ is given by

$$
0<k_{n}<\frac{a\left(1+t_{b}\right)^{2}}{(1+a)^{2} t_{b}} .
$$

When $k_{n}<1$, the spectrum response has two notches. When $k_{n}>1$, the spectrum response has a single notch. However, when the coupling between bus line and resonator is weaker than the transmittance in the resonator, i.e., $t_{b}>a\left(\kappa_{b}<1-\right.$ a), the optimum condition cannot be realized.

When $k_{n}<1$, the wavelength at which the transmittance of through port takes minima must satisfy the condition

$$
\cos \left(\beta_{n} L\right) \sim \sqrt{k_{n}}
$$

where $\beta_{n}$ is the propagation constant at notch wavelength. The wavelength of the notch varies depending on the coupling efficiency, even if the optical path lengths of resonators are identical. In addition, it should be noted that the notch wavelength is different from the wavelength satisfying $\cos (\beta L)=1$, which is supposed to be the resonant wavelength.

The through response is characterized by the attenuation at center wavelength $P_{t c}$, the attenuation at resonant wavelength $P_{t n}$, and the bandwidth of the notch $\Delta \lambda_{n}$, as shown in Fig. 7 .

1) Transmittance at Center Wavelength: The transmittance at center wavelength $P_{t c}$ is obtained from (9) by putting $\cos (\beta L)=1$, i.e.,

$$
P_{t c}=\frac{\left[(1+a) \sqrt{t_{b}}-\sqrt{a}\left(1+t_{b}\right) \sqrt{t_{r}}\right]^{2}}{\left[1+a t_{b}-2 \sqrt{a t_{b} t_{r}}\right]^{2}} .
$$

Using (10)-(13), $P_{t c}$ is rewritten as

$$
P_{t c}=\frac{A d_{+}\left(1-\sqrt{k_{n}}\right)^{2}}{1+A\left(\sqrt{k_{d}}-1\right)^{2}} .
$$

Fig. 8 shows the transmittance at center wavelength with $\kappa_{b}=0.2$. In the lossless case, the results for other values of $\kappa_{b}$ are almost similar to Fig. 8. At the optimum condition, i.e., $k_{n}=1$, then $P_{t c}=0$ can be obtained, even if resonators involve a loss. By increasing the discrepancy from the optimum condition, the attenuation at center wavelength gets worse. A large loss reduces the transmittance at center wavelength due to resonant enhancement. The weaker $\kappa_{b}$ is, the stronger is the 


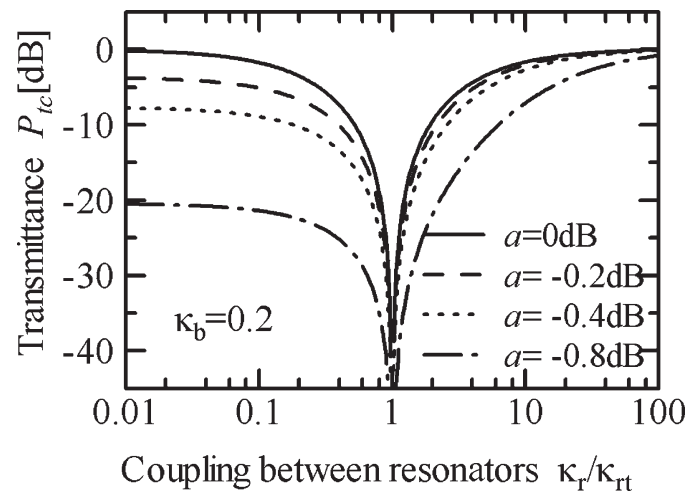

Fig. 8. Transmittance at center wavelength (through port).

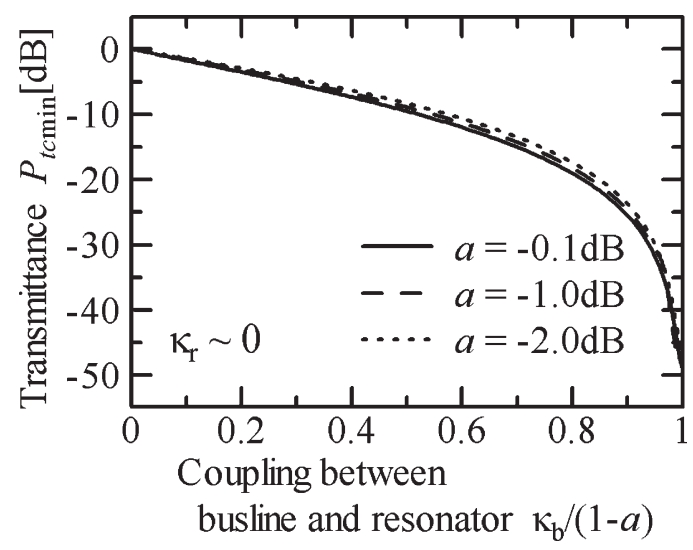

Fig. 9. Transmittance at center wavelength (through $\kappa_{r} \sim 0$ ).

resonant enhancement of loss. However, although the resonant enhancement effect depends on $\kappa_{b}$, the curve of $P_{t c}$ is slightly shifted downward compared with the curve shown in Fig. 8, except for the convergence to $0 \mathrm{~dB}$ as $\kappa_{r} / \kappa_{r t} \rightarrow \infty$. If the transmittance at the coupling region between bus line and resonator is larger than the transmittance per round in the resonator, i.e., if $t_{b}>a$, the optimum condition giving $P_{t c}=0$ cannot be realized, and $P_{t c}$ increases monotonically with the coupling between resonators. When $t_{b}<a$, i.e., there is an optimum condition, the minimum value of transmittance at center wavelength is zero. When $t_{b}>a$, the minimum value of transmittance at center wavelength $P_{t c \min }$ is obtained at $t_{r}=1$ and is given by

$$
P_{t c \min }=\frac{\left(\sqrt{a}-\sqrt{t_{b}}\right)^{2}}{\left(1-\sqrt{a t_{b}}\right)^{2}} .
$$

Fig. 9 shows the relation of (33). As shown in Fig. 9, if $t_{b} \gg$ $a\left(\kappa_{b} \ll 1-a\right)$, a deep notch cannot be obtained.

2) Notch Depth: When $k_{n}<1$, the notch becomes forked. Then the most attenuated wavelength satisfies $\cos (\beta L) \sim k_{n}$. The transmittance at notch $P_{t n}$ is obtained from (9) by putting $\cos (\beta L)=\sqrt{k_{n}}$ as

$$
P_{t n}=\frac{(1-a)^{2}\left[(1+a)^{2} t_{b}-a\left(1+t_{b}\right)^{2} t_{r}\right]}{\left(1-a t_{b}\right)^{2}\left[(1+a)^{2}-4 a t_{r}\right]}
$$

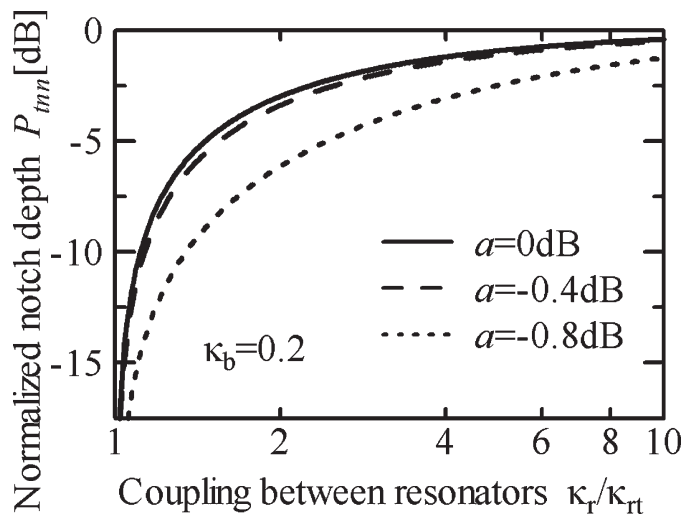

Fig. 10. Relation between notch depth and $\kappa_{r}$.

Using (10)-(13), $P_{t n}$ is rewritten as

$$
P_{t n}=\frac{A d_{-}\left(1-k_{n}\right)}{1+A\left(\sqrt{k_{d}}-\sqrt{k_{n}}\right)^{2}} .
$$

This equation represents the minimum transmittance in the case of $k_{n}<1$. When $k_{n}>1$ and $t_{b}>a$, the transmittance at center wavelength $P_{t c}$ is a minimum.

When resonators involve a loss, a small amount of light transmits to the through port, unless $k_{n}=1$. When the coupling between resonators is stronger than the optimum condition, the minimum transmittance is approximately independent of the coupling between resonators and is obtained as

$$
\left.P_{t n}\right|_{k_{n} \ll 1}=\frac{(1-a)^{2} t_{b}}{\left(1-a t_{b}\right)^{2}} .
$$

Equation (35) can be normalized by (36) as

$$
P_{t n n}=\frac{P_{t n}}{\left.P_{t n}\right|_{k_{n} \ll 1}}=\frac{\left(1-k_{n}\right)\left(1+t_{b}\right)^{2}}{\left[\left(1+t_{b}\right)^{2}-4 k_{n} t_{b}\right]} .
$$

This equation is independent of the loss in the resonator.

Fig. 10 shows the relation of (37). As shown in Fig. 10, the notch depth deepens at the optimum condition. When the coupling between resonators is stronger than the optimum condition, notch depth is saturated to the value of (36). If $t_{b}>a$, the notch depth deepens as the coupling between resonators decreases and is saturated to the value of (33). The propagation loss in the resonator reduces the transmittance at center wavelength by resonant enhancement. When $\kappa_{b}$ is weaker than 0.2 , which is often used in our experiment to obtain a clear filter response, the resonant enhancement effect is more stronger and the curve of $P_{t n n}$ shown in Fig. 10 is slightly shifted downward except for the convergence to $0 \mathrm{~dB}$ as $\kappa_{r} / \kappa_{r t} \rightarrow \infty$.

3) Notch Width: The bandwidth of the notch $\Delta \phi_{n}=$ $2\left(\beta_{3 \mathrm{~dB}} L-\beta_{c} L\right)$ is independent of the loss in the resonator in the range where the notch depth is sufficiently deep. Then the bandwidth of the notch is expressed by

$$
\Delta \phi_{n}=2 \cos ^{-1}\left(\sqrt{k_{n}}-\frac{1}{\sqrt{A}}\right) .
$$




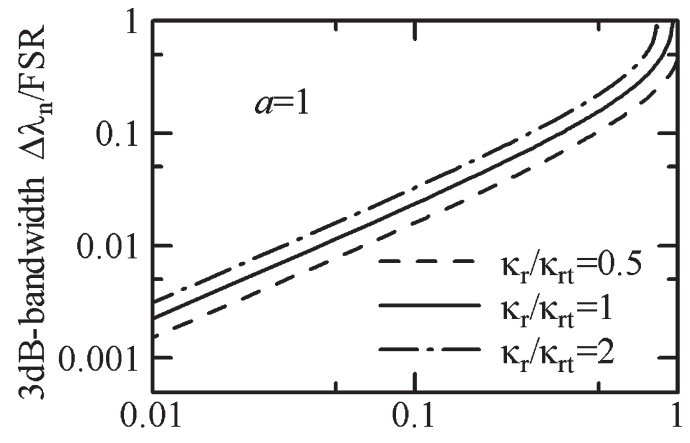

Coupling between busline and resonator $\kappa_{\mathrm{h}}$

Fig. 11. Relation between notch width and $\kappa_{r}$.

Using (10)-(13), $\Delta \phi_{n}$ is rewritten as

$$
\Delta \phi_{n}=2 \cos ^{-1}\left(\frac{\left(1+t_{b}\right) \sqrt{t_{r}}-\left(1-t_{b}\right) \sqrt{1-t_{r}}}{2 \sqrt{t_{b}}}\right)
$$

where $\beta_{c}$ and $\beta_{3 \mathrm{~dB}}$ are propagation constants at center wavelength and at the wavelength corresponding to $-3-\mathrm{dB}$ transmission, respectively. The $3-\mathrm{dB}$ bandwidth is $\Delta \lambda_{n}=$ $\left(2 \pi n L / \lambda_{c}^{2}\right) \Delta \phi_{n}$. Fig. 11 shows the 3 -dB bandwidth against the coupling coefficient $\kappa_{b}$. When the resonator involves a loss, the bandwidth is slightly broadened. When $k_{n}=1$

$$
\Delta \phi_{n}=\cos ^{-1}\left(1-\frac{\left(1-t_{b}\right)^{2}}{2 \sqrt{t_{b}}\left(1+t_{b}\right)}\right) .
$$

Compared with a single ring resonator, the notch depth of a single ring resonator $\Delta \phi_{n s}$ is given by

$$
\Delta \phi_{n s}=\cos ^{-1}\left(1-\frac{\left(1-t_{b}\right)^{2}}{2 t_{b}}\right) .
$$

Therefore, $\Delta \phi_{n s} \sim \sqrt{2} \Delta \phi_{n}$ for the same value of $t_{b}$.

\section{B. Drop Port Response}

The transmittance of the drop port is expressed by the discrepancy from the optimum condition as

$$
t_{r}=k_{d} \frac{4 a t_{b}}{\left(1+a t_{b}\right)^{2}}
$$

where $k_{d}$ is the ratio of $t_{r}$ and $t_{r d}$. Since $0<t_{r}<1, k_{d}$ is in the range

$$
0<k_{d}<\frac{\left(1+a t_{b}\right)^{2}}{4 a t_{b}} .
$$

When $k_{d}=1$, the peak is maximally flat, and this is the so-called Butterworth filter characteristics. When $k_{d}<1$, the spectrum response has two peaks, and this is the so-called Chebyshev filter characteristics [10]. When $k_{d}>1$, the spectrum response has a single peak.

Peak wavelengths satisfy the condition

$$
\cos \left(\beta_{p} L\right)=\sqrt{k_{d}}
$$

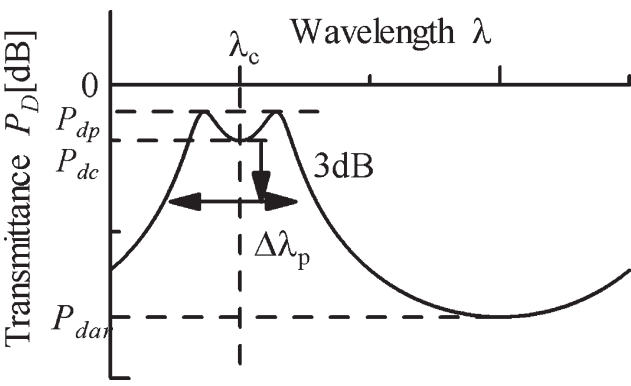

Fig. 12. Spectrum shape of drop port response.

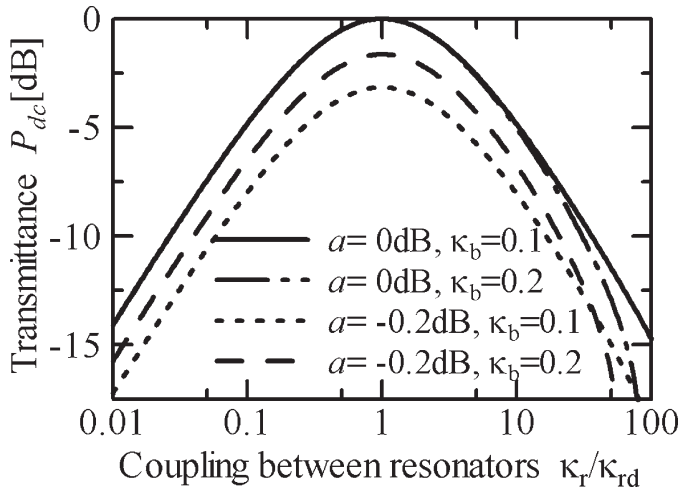

Fig. 13. Transmittance at center wavelength (drop port).

where $\beta_{p}$ is the propagation constant at peak wavelength. Peak wavelengths vary with coupling efficiency, even if the optical path lengths of resonators are identical.

The drop port response is characterized by the transmittance at center wavelength $P_{d c}$, the peak transmittance $P_{d p}$, bandwidth of the peak $\Delta \lambda_{p}$, and the attenuation at anti-resonant wavelength $P_{d a r}$, as shown in Fig. 12.

1) Transmittance at Center Wavelength: The transmittance at center wavelength $P_{d c}$ is obtained from (14) by setting $\cos (\beta L)=1$ as

$$
P_{d c}=\frac{a\left(1-t_{b}\right)^{2}\left(1-t_{r}\right)}{\left[\left(1+a t_{b}\right)-2 \sqrt{a t_{b} t_{r}}\right]^{2}} .
$$

Fig. 13 shows the transmittance at center wavelength. When resonators are lossless and the coupling efficiencies are set to the optimum condition, the transmittance at center wavelength is unity. When this condition is not satisfied, the transmittance at center wavelength is reduced from unity. For example, when the resonator has a loss of $0.2 \mathrm{~dB} /$ round, the insertion loss is as high as $3.1 \mathrm{~dB}$ when $\kappa_{b}=0.2$ and $\kappa_{r}$ is optimized $\left(\kappa_{r}=\kappa_{r} d\right)$. If $\kappa_{r}$ is not optimized, the insertion loss is larger than $3.1 \mathrm{~dB}$. In the lossless case, $P_{d c}$ is independent of $\kappa_{b}$. When the resonator involves a loss, a smaller $\kappa_{b}$ reduces $P_{d c}$.

2) Peak Transmittance: When $k_{d}<1$, the peak splits into two in the same way as the case of $k_{t}<1$ in the through port response. Then the wavelength giving the largest transmittance satisfies $\cos (\beta L)=\sqrt{k_{d}}$. The transmittance at peak wavelength $P_{d p}$ is obtained from (14) as follows, assuming $\cos (\beta L)=\sqrt{k_{d}}$, i.e.,

$$
P_{d p}=p
$$




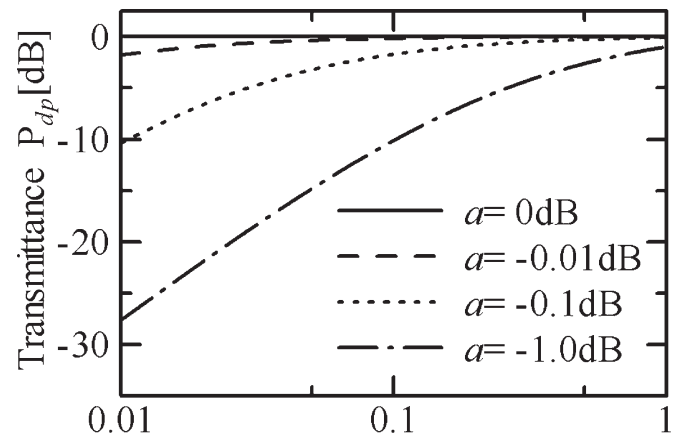

Coupling between busline and resonator $\kappa_{\mathrm{b}}$

Fig. 14. Relation between peak transmittance and $\kappa_{r}$.

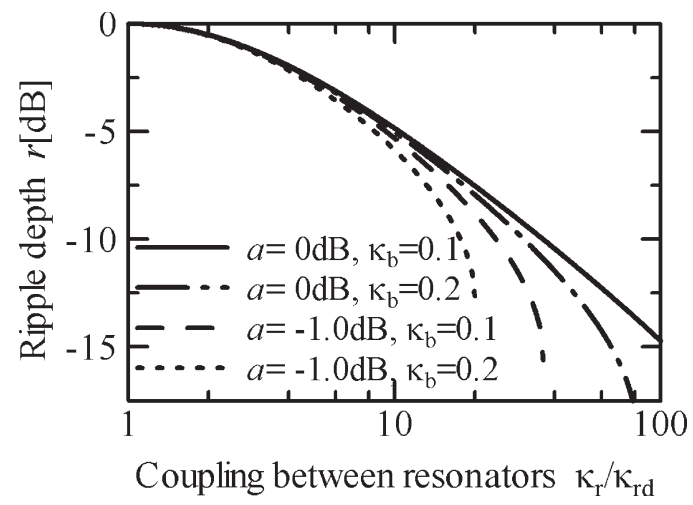

Fig. 15. Relation between ripple depth and $\kappa_{r}$.

where $p$ is defined by (15). The peak transmittance is independent of the coupling between resonators $\kappa_{r}$. Fig. 14 shows the peak transmittance against the coupling efficiency $\kappa_{b}$.

3) Ripple Depth: When $k_{d}<1$, the drop response has two peaks. Then the ripple depth is defined by $P_{d c} / P_{d p}$, i.e., the ratio of the transmittance at center wavelength to the transmittance at peak wavelength. This can be derived from (45) and (46) as

$$
r \equiv \frac{P_{d c}}{P_{d p}}=\frac{\left(1-a t_{b}\right)^{2}\left(1-t_{r}\right)}{\left[\left(1+a t_{b}\right)-2 \sqrt{a t_{b} t_{r}}\right]^{2}}
$$

Fig. 15 shows the ripple depth against the coupling efficiency between the resonators $\kappa_{r}$. By increasing $\kappa_{r}$ from $\kappa_{r t}$, ripple depth is increased. Ripple depth is independent of the loss and coupling between bus line and resonator around the optimum condition.

The transmittance at the coupling region between resonators $t_{r r}$, which involve the ripple depth $r=P_{d c} / P_{d p}$, is given by

$$
t_{r r}=\frac{\left[2 r \sqrt{a t_{b}}\left(1+a t_{b}\right)-\sqrt{1-r}\left(1-a t_{b}\right)^{2}\right]^{2}}{\left[\left(1-a t_{b}\right)^{2}+4 r a t_{b}\right]^{2}} .
$$

Fig. 16 shows the optimum coupling efficiency between resonators $\kappa_{r}$ against that between the bus line and the resonator $\kappa_{b}$ with the parameter of ripple depth $r$.

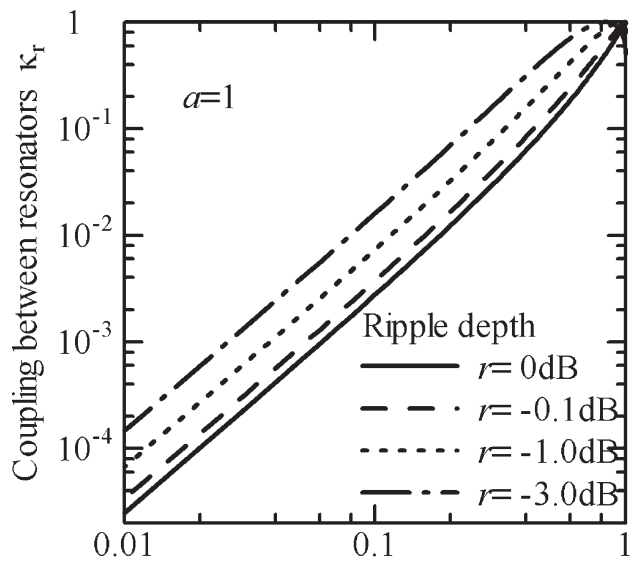

Coupling between busline and resonator $\kappa_{\mathrm{b}}$

Fig. 16. Optimum condition when drop port response involves ripple.

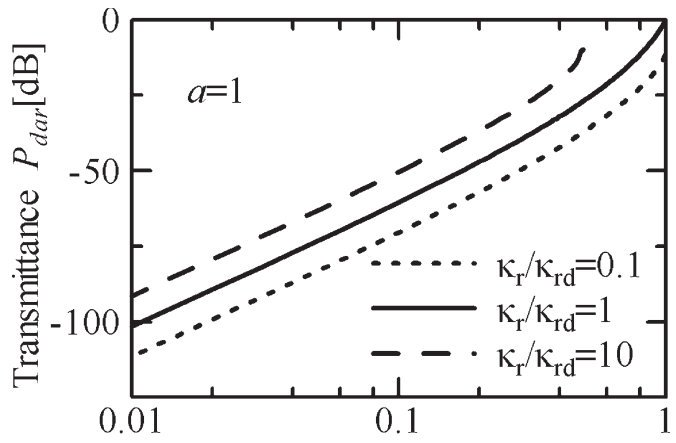

Coupling between busline and resonators $\kappa_{b}$

Fig. 17. Transmittance at anti-resonant wavelength.

4) Transmittance at Anti-Resonant Wavelength: At antiresonant wavelength corresponding to $\cos (\beta L)=-1$, the transmittance to the drop port is minimum, i.e.,

$$
P_{d a r}=\frac{a\left(1-t_{b}\right)^{2}\left(1-t_{r}\right)}{\left[\left(1+a t_{b}\right)+2 \sqrt{a t_{b} t_{r}}\right]^{2}} .
$$

Fig. 17 shows the transmittance at anti-resonant wavelength against the coupling efficiency between the bus line and the resonator $\kappa_{b} . P_{d a r}$ is almost independent of the loss in the resonator.

5) Peak Width: The bandwidth of the peak $\Delta \phi_{p}=2\left(\beta_{b} L-\right.$ $\left.\beta_{c} L\right)$ is expressed by

$$
\Delta \phi_{p}(b)=2 \cos ^{-1}\left(\sqrt{k_{d}}-\sqrt{\frac{1-b+A\left(1-\sqrt{k_{d}}\right)^{2}}{b A}}\right)
$$

where $\beta_{b}$ is the propagation constant for the case where the transmittance to drop port is $b P_{d c}$, and $b$ is the band level. The $-3-\mathrm{dB}$ bandwidth is rewritten by substituting $b=1 / 2$ into (50) as

$$
\Delta \phi_{p}=2 \cos ^{-1}\left(\sqrt{k_{d}}-\sqrt{\frac{1+2 A\left(1-\sqrt{\left.k_{d}\right)^{2}}\right.}{A}}\right) .
$$




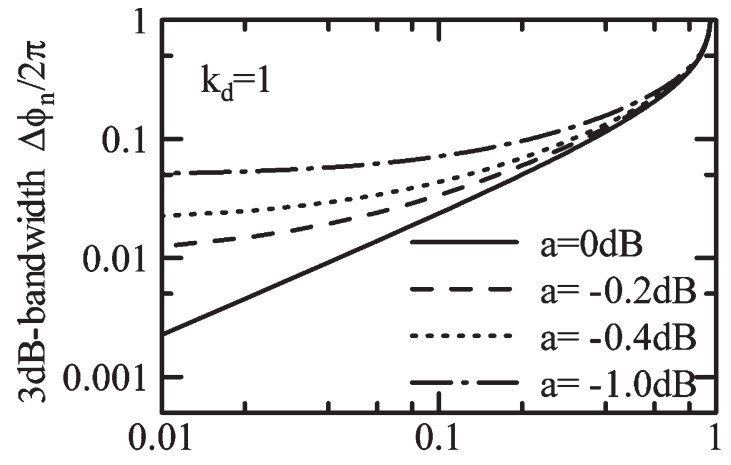

Coupling between busline and resonators $\kappa_{\mathrm{b}}$

Fig. 18. Relation between bandwidth and $\kappa_{r}$.

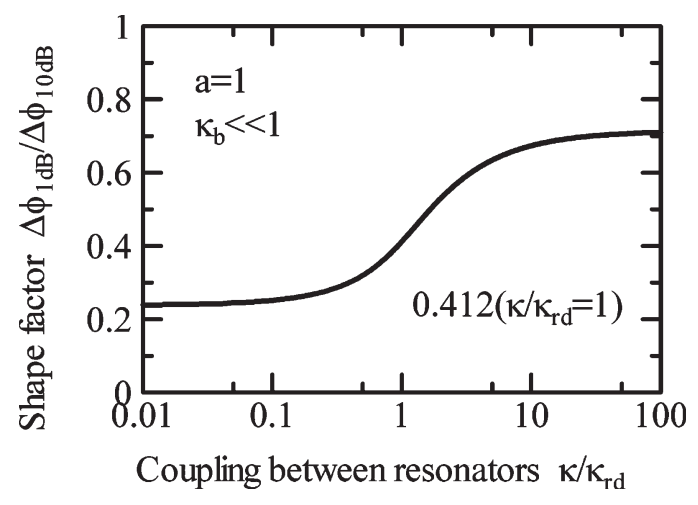

Fig. 19. Relation between shape factor and $\kappa_{r}$.

In the lossless case, the bandwidth varies against the coupling efficiency $\kappa_{b}$ as shown in Fig. 11 because the notch width is equal to the bandwidth of the drop port response. When the resonator involves loss, the behavior of the bandwidth is shown in Fig. 18. By increasing loss, the bandwidth is broadened.

6) Shape Factor: The shape factor $\left(\Delta \phi_{p}\left(\begin{array}{ll}-1 & \mathrm{~dB}\end{array}\right) /\right.$ $\left.\Delta \phi_{p}(-10 \mathrm{~dB})\right)$ denotes the degree of the box-like spectrum shape. Fig. 19 shows the shape factor against the coupling efficiency between resonators. When the coupling efficiency between resonators is strong, the shape factor is large. However, increasing $\kappa_{r}$ increases the ripple.

When the resonator is lossless and the coupling efficiency is optimum and weak, i.e., $a=1$ and $k_{d}=1$, then $t_{b}$ approaches unity. Thus, we obtain

$$
\begin{aligned}
\lim _{t_{b} \rightarrow 1} \frac{\Delta \phi_{p}\left(b_{1}\right)}{\Delta \phi_{p}\left(b_{2}\right)} & =\left[\frac{\left(1-b_{1}\right) b_{2}}{b_{1}\left(1-b_{2}\right)}\right]^{\frac{1}{4}} \\
\text { Shape factor } & =\lim _{t_{b} \rightarrow 1} \frac{\Delta \phi_{p}(-1 \mathrm{~dB})}{\Delta \phi_{p}(-10 \mathrm{~dB})} \sim 0.412 .
\end{aligned}
$$

In the same way, the shape factor of the single ring resonator is given by

$$
\text { Shape factor }_{\text {single }}=\left[\frac{\left(1-10^{-0.1}\right) 10^{-1}}{10^{-0.1}\left(1-10^{-1}\right)}\right]^{\frac{1}{2}} \sim 0.170 .
$$

The shape factor of the $n$ th-order series-coupled microring resonator with optimum coupling condition is represented as

$$
\text { Shape } \text { factor }_{n}=\left[\frac{\left(1-10^{-0.1}\right) 10^{-1}}{10^{-0.1}\left(1-10^{-1}\right)}\right]^{\frac{1}{2 n}} \text {. }
$$

The shape factor of the Gaussian filter is about 0.316. Then the second-order series-coupled ring resonator has a box-like spectrum compared to the Gaussian filter.

\section{CONCLUSiON}

The optimum conditions of coupling efficiencies of a double series-coupled ring resonator are derived when ring resonators involve loss. In the condition given by (17), no output power is transmitted to the through port at center wavelength. In the condition given by (24), the peak of the transmittance to the drop port is maximally flat. In the condition given by (48), the peak has a ripple and the ripple depth is $r$.

In the case when the resonator is lossless, (17) and (24) are identical. Then the through port response has a sharp notch and the drop port response has a flat peak.

However, when the resonator involves loss, the optimum condition is different from the lossless case and the sharp notch, and the flattened peak cannot be achieved simultaneously. The crosstalk between through port and drop port responses is deteriorated by the loss in the resonator. To obtain the maximally flat peak of drop port response and the deep notch of through port response simultaneously, the coupling efficiency should be stronger than the loss in resonator, i.e., $\kappa_{b} \ll 1-a$.

\section{REFERENCES}

[1] S. Suzuki, K. Shuto, and Y. Hibino, "Integrated optic ring resonators with two stacked layers of silica waveguides on Si," IEEE Photon. Technol. Lett., vol. 4, no. 11, pp. 1256-1258, Nov. 1992.

[2] B. E. Little, S. T. Chu, J. Forsi, G. Steinmeyer, E. Thoen, H. A. Haus, E. P. Ippen, L. Kimerling, and W. Grrene, "Microresonators for integrated optical devices," Opt. Photon. News, vol. 9, no. 12, pp. 32-33, Dec. 1998.

[3] S. Yamagata, T. Kato, and Y. Kokubun, "Non-blocking wavelength channel switch using TO effect of double series coupled microring resonator," Electron. Lett., vol. 41, no. 10, pp. 593-595, May 2005.

[4] B. E. Little, S. T. Chu, H. A. Haus, J. Foresi, and J. P. Laine, "Microring resonator channel dropping filters," J. Lightw. Technol., vol. 15, no. 6, pp. 998-1005, Jun. 1997.

[5] A. Yariv, "Universal relations for coupling of optical power between microresonators and dielectric waveguides," Electron. Lett., vol. 36, no. 4, pp. 321-322, Feb. 2000.

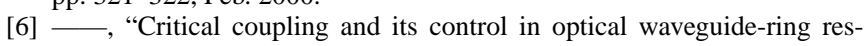
onator systems," IEEE Photon. Technol. Lett., vol. 14, no. 4, pp. 483-485, Apr. 2002.

[7] G. Barbarossa, A. M. Matteo, and M. N. Armenise, "Theoretical analysis of triple-coupler ring-based optical guided-wave resonator," J. Lightw. Technol., vol. 13, no. 2, pp. 148-157, Feb. 1995.

[8] R. Orta, P. Savi, R. Tascone, and D. Trinchero, "Synthesis of multiplering-resonator filters for optical systems," IEEE Photon. Technol. Lett., vol. 7, no. 12, pp. 1447-1449, Dec. 1995.

[9] C. K. Madsen and J. H. Zhao, "A general planar waveguide autoregressive optical filter," J. Lightw. Technol., vol. 14, no. 3, pp. 437-447, Mar. 1996.

[10] A. Melloni and M. Martinelli, "Synthesis of direct-coupled-resonators bandpass filters for WDM systems," J. Lightw. Technol., vol. 20, no. 2, pp. 296-303, Feb. 2002. 


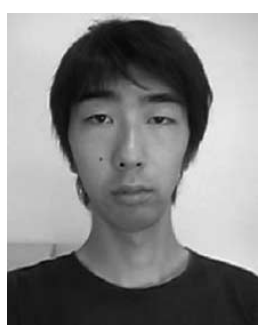

Tomoyuki Kato (S'05) was born in Saitama, Japan, on January 3, 1979. He received the B.E. and M.E degrees in electrical and computer engineering from Yokohama National University, Yokohama, Japan, in 2001 and 2003, respectively.

His main interests include optical waveguide-type functional devices.

Mr. Kato is a member of the Institute of Electronics, Information and Communication Engineers (IEICE).

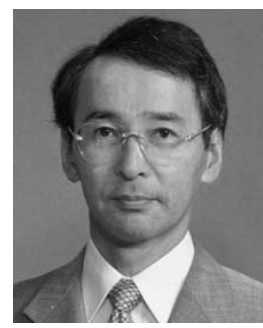

Yasuo Kokubun (M'85-SM'05) was born in Fukushima, Japan, on July 7, 1952. He received the B.E. degree from Yokohama National University, Yokohama, Japan, in 1975 and the M.E. and Dr.Eng. degrees from Tokyo Institute of Technology, Tokyo, Japan, in 1977 and 1980, respectively.

After working for the Research Laboratory of Precision Machinery and Electronics, Tokyo Institute of Technology, as a Research Associate from 1980 to 1983, he joined the Yokohama National University as an Associate Professor in 1983 and is currently a Professor at the Department of Electrical and Computer Engineering. His current research is in integrated photonics, especially waveguide-type functional devices and three-dimensional integrated photonics. From 1984 to 1985 he was a Visiting Researcher at AT\&T Bell Laboratories, Holmdel, NJ, and was engaged in a novel waveguide on semiconductor substrate (ARROW) for integrated optics. From 1996 to 1999, he served as the Project Leader of the "three-dimensional microphotonics" project at the Kanagawa Academy of Science and Technology.

Prof. Kokubun is a member of the Japan Society of Applied Physics, and the Optical Society of America (OSA). 